\title{
Effect of varicocelectomy treatment on spermatogenesis and apoptosis via the induction of heat shock protein 70 in varicocele-induced rats
}

\author{
JIN-ZHUO NING*, TING RAO*, FAN CHENG, WEI-MIN YU, YUAN RUAN, \\ RUN YUAN, SHAO-MING ZHU, YANG DU and CHENG-CHENG XIAO \\ Department of Urology, Renmin Hospital of Wuhan University, Wuhan, Hubei 430060, P.R. China
}

Received October 17, 2016; Accepted June 13, 2017

DOI: $10.3892 / \mathrm{mmr} .2017 .7239$

\begin{abstract}
In the current study, the hypothesis that testicular varicocelectomy improves spermatogenesis and attenuates apoptosis via the induction of heat shock protein 70 (Hsp70) in a rat model of varicocele was investigated. Adult male Wistar rats $(\mathrm{n}=75)$ were randomly divided into 5 groups of 15 each: Control, sham, varicocele, varicocelectomy, and varicocelectomy plus Quercetin. A total of 6 weeks after the varicocelectomy, the left testis of all rats was removed for subsequent examination. Histological changes were compared between the groups. The expression of Hsp70 and apoptosis-associated indicators were evaluated based on immunohistochemical, western blot and mRNA expression analyses. Compared with the varicocele group, the varicocelectomy group exhibited a markedly reduced Bcl-2-associated $\mathrm{X}$ protein/B-cell lymphoma 2 (Bax/Bcl-2) ratio, and had a decreased expression of caspase-9, cytochrome $c$ (cyt $c$ ) and caspase-3 through the intrinsic signal transduction pathways. Quercetin treatment inhibited the protective effects of varicocelectomy. The expression of Hsp70 was increased in the varicocele group which was further elevated by the varicocelectomy. These results indicated that varicocelectomy can reduce the Bax/Bcl-2 ratio, and decrease the levels of caspase- 9 , cyt $c$ and caspase-3 via the mitochondrial signal transduction pathway. Such protective effects on left testis spermatogenesis and against apoptosis may be due to the induction of Hsp70. The findings of the present study suggested that varicocelectomy has a clear advantage in protecting testicular function and ameliorating spermatogenic cells apoptosis.
\end{abstract}

Correspondence to: Dr Fan Cheng, Department of Urology, Renmin Hospital of Wuhan University, 238 Jiefang Road, Wuhan, Hubei 430060, P.R. China

E-mail: chenfan_93@126.com

${ }^{*}$ Contributed equally

Key words: varicocelectomy, varicocele, heat shock protein 70 , spermatogenesis, apoptosis

\section{Introduction}

Varicocele is defined as the abnormal expansion, elongation and tortuosity of the spermatic vein, which has been considered the main cause of male infertility (1). The pathogenesis of testicular dysfunction or the mechanism of infertility caused by varicocele has yet to be defined. Testicular dysfunction may be associated with many factors, such as microcirculation disturbance of the testis, vasoactive substance reflux, oxidative stress, nitric oxide (NO), hypoxia, hyperthermia or apoptosis (2). The surgical procedure, varicocelectomy, can enhance spermatogenesis, improve the quality of semen and prevent apoptosis of cells in the testis, making it an effective varicocele treatment (3). Apoptosis is a normal physiological phenomenon that serves an important role in maintaining the homeostasis of the body $(4,5)$. Normally, during spermatogenesis, $\sim 25-75 \%$ of spermatogenic cell undergo apoptosis, maintaining the normal reproductive function through regulation of the apoptosis signal transduction pathway. According to a previous study, apoptosis of spermatogenic cells is increased in a rat model of varicocele, which leads to impaired testicular function and male infertility (6). Apoptosis of spermatogenic cells in rat testis is decreased after varicocelectomy treatment (7). As apoptosis is extremely important in testicular damage and male infertility induced by the varicocele, the ideal treatment is to target the associated apoptotic pathways.

Heat shock proteins (HSPs) are a group of highly conserved protein molecules that exist in almost all organisms, ranging from prokaryotes to eukaryotes (8). Under conditions of ischemia, hypoxia, cellular energy depletion, metabolic products, oxidative stress and inflammation, HSPs can be induced to serve their regulatory roles (9). Hsp70 has been widely and thoroughly studied. Hsp70 is expressed at low levels under physiological conditions and has specific regulatory effects on cell growth, development, differentiation and cell death. It is reported that Hsp70 regulates the different stages of spermatogenesis and that its abnormal expression of may interfere with male fertility $(10,11)$.

The current study investigated the hypothesis that varicocelectomy may increase the expression of Hsp70 via the mitochondrial signal transduction pathway, thereby reducing apoptosis and protecting spermatogenesis. Furthermore, 
whether varicocelectomy reduces apoptosis in a rat experimental varicocele model was examined. In this study, hematoxylin and eosin (H\&E) staining, terminal deoxynucleotidyl-transferase-mediated dUTP nick end labelling (TUNEL) assays, immunohistochemistry, western blot analysis and reverse transcription-quantitative polymerase chain reaction (RT-qPCR) analysis of the left testes were performed to test the above hypotheses.

\section{Materials and methods}

Animals. The present study involved 75 adult male Wistar rats (8-10 weeks), weighing 200-250 g, that were provided by the Hubei Center for Disease Control (Wuhan, China). Prior to the experiment, rats received free access to food and water in a 12-h light/dark room with a constant temperature $\left(22 \pm 2^{\circ} \mathrm{C}\right)$ and (40-70\%) humidity. All experiments were approved by the Animal Experimental Ethics Committee of Wuhan University (Wuhan, China).

Experimental grouping and surgical procedures. Rats were randomly divided into 5 groups with 15 rats in each group: Control, sham, varicocele, varicocelectomy, and varicocelectomy plus Quercetin. The surgery was performed on rats that were anesthetized with intraperitoneal administration of $2 \%$ sodium phenobarbital (50 mg/kg; Beijing Propbs Biotechnology. Co., Ltd.). In the control group, rats did not undergo surgery. In the sham group, an abdominal median incision was performed. Following exposure, the left renal vein was only separated, without ligation, and the incision was sutured with 4-0 silk. In the varicocele group, the left renal vein was carefully separated from the inside of the adrenal vein and the spermatic vein, and a smooth metal rod $(0.5-\mathrm{mm}$ diameter) was placed parallel to the left renal vein. The vein and metal rod were ligated with 4-0 silk, which reduced the vein diameter to approximately half its original diameter. The metal rod was then pulled out, and the left renal vein was reversibly constricted, as previously described (12), including the following steps: Abnormal expansion, elongation and tortuosity of the left renal and spermatic vein; the diameter of the left renal vein greater than $1 \mathrm{~mm}$; and no pathological lesion in the left kidney, such as atrophy (Fig. 1).

In the varicocelectomy group, 4 weeks after the successful establishment of the varicocele model in rats, varicocelectomy was performed by a left lower abdominal incision.

In the varicocelectomy and Quercetin group, in addition to the procedures performed in the varicocelectomy group, Quercetin (10 mg/kg/day) (Sigma-Aldrich; Merck KGaA, Darmstadt, Germany) was injected intraperitoneally for 6 weeks following ligation of the internal spermatic cord veins.

Preservation of testis. A total of 6 weeks after the varicocelectomy, all left testes of the rats were removed, dissected of associated adipose tissue and fascia attached to the surface of testes, and left to dry on filter paper after washing with saline. The testes were then cut in half, with one half fixed in $4 \%$ paraformaldehyde for histological examination. The other half was immediately frozen and stored at $-80^{\circ} \mathrm{C}$ for later analysis.

$H \& E$ staining. After fixation in $4 \%$ paraformaldehyde, the half testes were embedded in paraffin and were cut to $5-\mu \mathrm{m}$

\section{Successful modeling of varicocele}

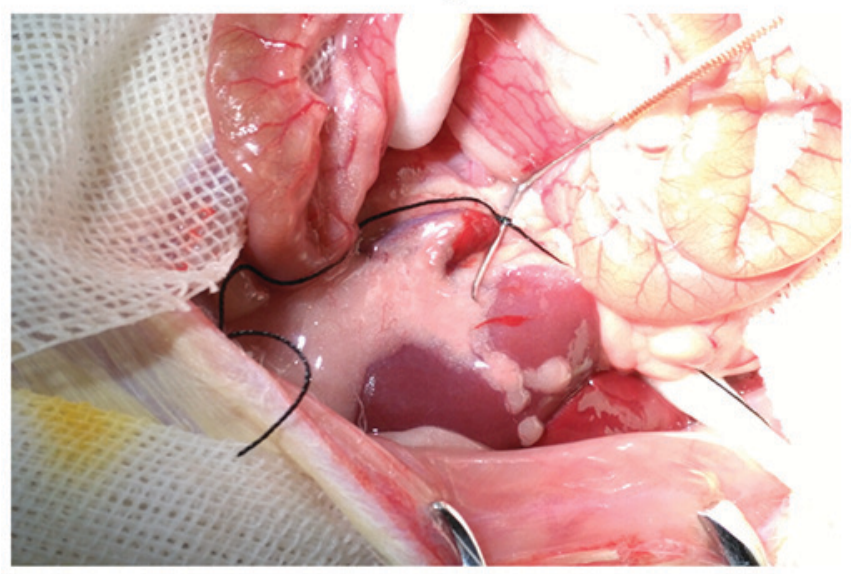

Figure 1. Successful modeling of varicocele.

sections. After routinely dewaxing and hydration, sections were stained with hematoxylin for $5 \mathrm{~min}$ and eosin for $2 \mathrm{~min}$ at the room temperature. prior to sealing.

TUNEL assay. To observe spermatogenic cell apoptosis in the testes, an in situ apoptosis detection kit (Promega Corporation, Madison, WI, USA) was used and a terminal deoxynucleotidyl transferase dUTP nick end labeling (TUNEL) assay was performed, Briefly, the rat testis tissue was fixed in $4 \%$ paraformaldehyde/PBS solution ( $\mathrm{pH} 7.4$ ) at $4^{\circ} \mathrm{C}$ overnight and then placed into $70 \%$ ethanol at $20^{\circ} \mathrm{C}$ for $24 \mathrm{~h}$. Following washing 3 times with PBS, the samples were immersed in a permeabilization buffer for $15 \mathrm{~min}$. Subsequently, they were incubated with $50 \mathrm{ml}$ reaction buffer (TdT Enzyme $5 \mathrm{ml}+$ Labeling Safe Buffer $45 \mathrm{ml}$ ) at $37^{\circ} \mathrm{C}$ for $90 \mathrm{~min}$. The nuclei of the positive cells were stained as yellow brown or dark brown, observed under a Zeiss LSM 510 confocal laser scanning microscope (Zeiss AG, Oberkochen, Germany). From each section, 10 fields were randomly selected, and the number of positive cells were counted in order to calculate the apoptosis index.

Immunohistochemistry. The expression of Hsp70, Bcl-2-associated X protein (Bax) and caspase-3 was analyzed by immunohistochemical staining. Antibodies were purchased from Santa Cruz Biotechnology, Inc. (Dallas, TX, USA): i) Hsp70, a mouse monoclonal antibody (sc-32239; 1:50); ii) Bax, a rabbit polyclonal antibody (sc-493; 1:25); and iii) caspase-3, a rabbit polyclonal antibody (sc-7148; 1:100). All steps were performed according to the manufacturer's protocol and the results were observed with an Olympus BX50 light microscope (Olympus Corporation, Tokyo, Japan).

Western blot analysis. Testis tissue proteins from both groups were extracted with RIPA lysis buffer (P0013B; Beyotime Institute of Biotechnology, Haimen, China) and quantified using a Bicinchoninic Acid assay (K763-KIT; Amresco, LLC, Solon, OH, USA). Equivalents of protein samples (40 $\mu \mathrm{g} / \mathrm{lane})$ were separated by $10 \%$ sodium dodecyl sulfate-polyacrylamide gel electrophoresis and transferred onto a nitrocellulose membrane. The membrane was blocked with TBS/Tween-20 (TBST) buffer with 5\% non-fat milk and then incubated at $4{ }^{\circ} \mathrm{C}$ 


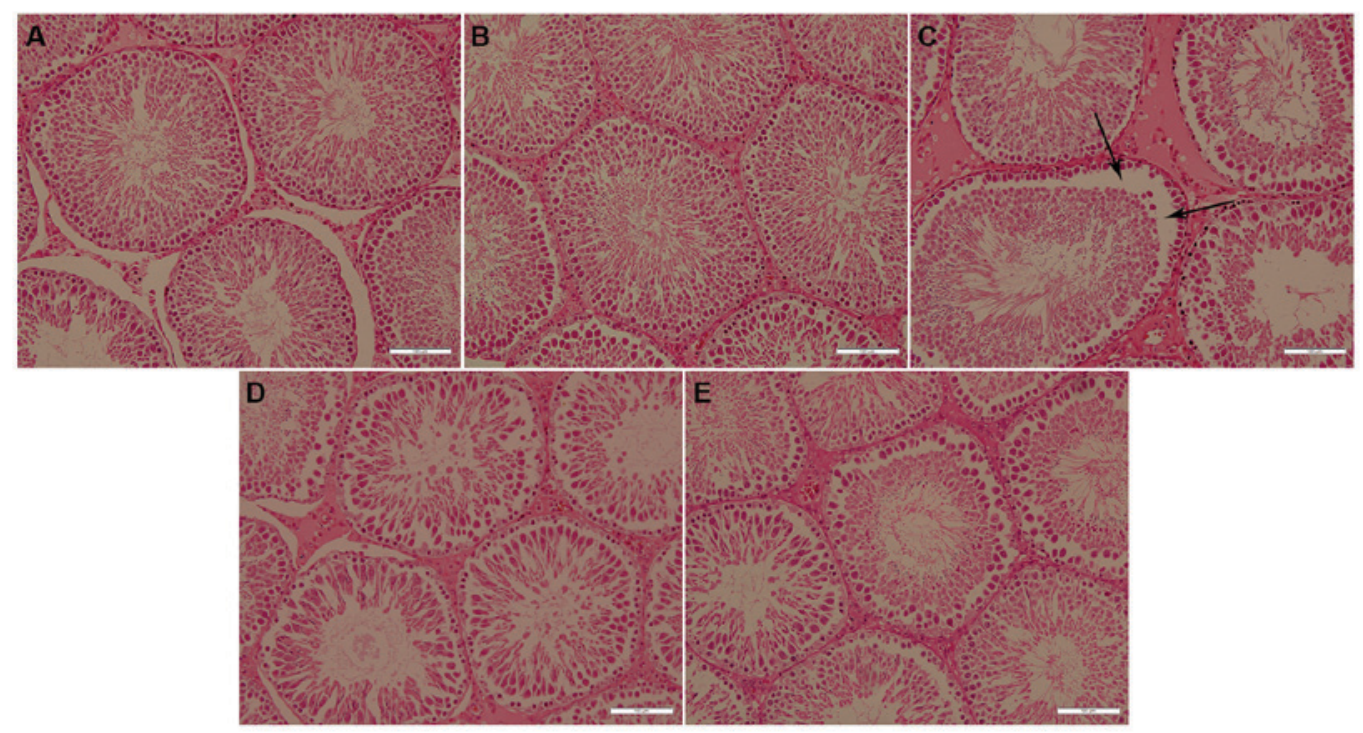

Figure 2. Testis tissues in the five groups used in the present study were analyzed using the hematoxylin and eosin stain (magnification, $\mathrm{x} 200$ ). Representative photomicrographs of rat testis tissue in the (A) control, (B) sham, (C) varicocele: damage of testicular spermatogenic function (arrows), (D) varicocelectomy and (E) varicocelectomy and Quercetin groups.

with the following primary antibodies: Bax (sc-493; 1:500), B-cell lymphoma (Bcl-2; sc-7382; 1:1,000), cytochrome $c$ (cyt $c ; 1: 2,000$ dilution; cat. no. Santa Cruz Biotechnology, Inc.) and cleaved caspase-3 (sc-13156; 1:1,000; all from Santa Cruz Biotechnology, Inc.) at $4^{\circ} \mathrm{C}$ overnight. Following rinsing twice with TBST, the membranes were incubated with secondary goat anti-rabbit antibodies conjugated with horseradish peroxidase (LK2001/LK2003; 1:100; Sungene Biotech, Co., Ltd., Tianjin, China) at room temperature for $1 \mathrm{~h}$. All specific bands were visualized using an enhanced chemiluminescence system (Pierce; Thermo Fisher Scientific Inc., Waltham, MA, USA). Optical densities were analyzed using the ImageJ software version $1.48 \mathrm{u}$ (National Institutes of Health, Bethesda, MD, USA).

$R T-q P C R$. Total RNA was extracted from the rat testis tissue samples using TRIzol reagent (Invitrogen; Thermo Fisher Scientific Inc.), and the RNA concentration was measured by spectrophotometry. First-strand cDNA was synthesized using a cDNA synthesis kit (Promega Corporation, Madison, WI, USA) according to the manufacturer's protocol. Subsequently, RT-qPCR was applied to the SYBR-Green mix kit (Applied Biosystems; Thermo Fisher Scientific Inc.). The primers for Hsp70, caspase-3 and caspase-9 were designed as follows: Hsp70 forward, 5'-ATGCTTCAGACCTCCCTT-3' and reverse, 5'-CTCCACCAACTATCTCCACT-3'; caspase-3 forward, 5'-TGGACTGCGGTATTGAGACA-3' and reverse, 5'-GCGCAAAGTGACTGGATGAA-3'; caspase-9 forward, 5'-CAAGAAGAGCGGTTCCTGGT-3' and reverse, 5'-CAG AAACAGCATTGGCGACC-3'; GAPDH was used as a housekeeping gene. The data were measured as a ratio of each mRNA relative to GAPDH mRNA (forward, 5'-ACAGCAACA GGGTGGTGGAC-3' and reverse, 5'-TTTGAGGGTGCAGCG AACTT-3'). PCR was performed with 40 cycles of $94^{\circ} \mathrm{C}$ for $30 \mathrm{sec}$, followed by $56^{\circ} \mathrm{C}$ for $30 \mathrm{sec}$ and $72^{\circ} \mathrm{C}$ for $25 \mathrm{sec}$, using the ABI 7900 Real-Time PCR system (Applied Biosystems; Thermo Fisher Scientific Inc., Waltham, MA, USA).
Statistical analysis. All data were presented as the mean \pm standard deviation. Statistical analysis was conducted using SPSS version 17.0 (SPSS Inc., Chicago, IL, USA). The means were compared using one-way analysis of variance followed by the Student-Newman-Keuls test for the different groups. $\mathrm{P}<0.05$ was considered to indicate a statistically significant difference. All experiments were performed at least three times.

\section{Results}

$H \& E$ staining. The left testis tissue sections were stained demonstrating that the spermatogenic cells within the seminiferous tubules were arranged in order and that there was sperm in the lumen in the control and sham groups. However, the testes in the varicocele group exhibited significant damage of testicular spermatogenic function, such as extensive seminiferous epithelium injury, the appearance of vacuoles, and a disorderly distribution of spermatogenic cells. There were fewer spermatogenic cells and seminiferous epithelium changes in the varicocelectomy group than in the varicocele group, while Quercetin inhibited the protective effects significantly (Fig. 2).

TUNEL assays. The results demonstrated rare and scattered TUNEL-positive spermatogenic cells in the control and sham groups. In the varicocele group, TUNEL-positive cells were widely observed, and there was an increase compared with the control groups. After varicocelectomy, the number of TUNEL-positive spermatogenic cells was reduced compared with the varicocele group. However, this effect was reversed by Quercetin (Fig. 3).

Immunohistochemistry. Hsp70, Bax and caspase-3 were evaluated using immunohistochemical techniques. Hsp70, Bax and caspase-3-positive cells were rarely observed in the rat testes from the control and sham groups. However, in the varicocele 

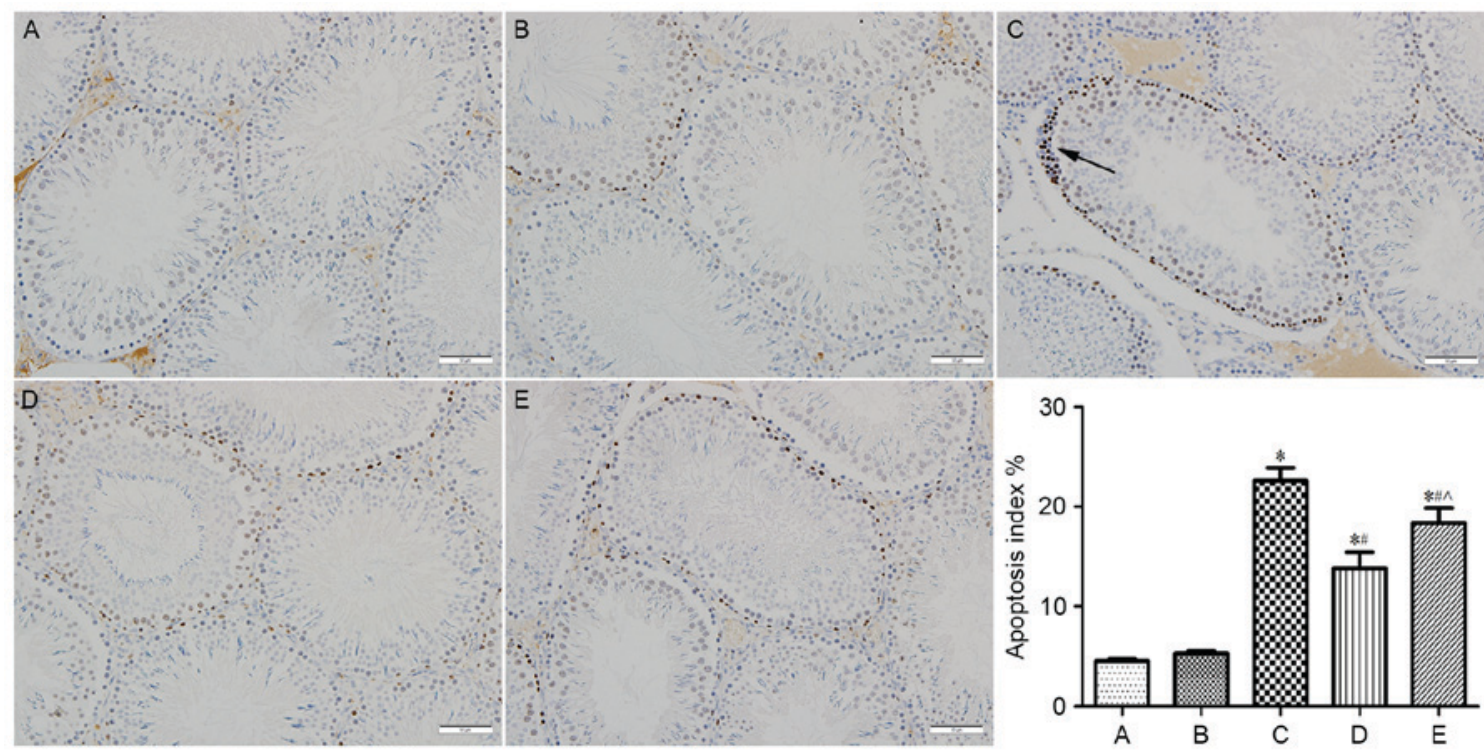

Figure 3. Spermatogenic cells apoptosis in the five groups was studied using the TUNEL assay (magnification, x200). TUNEL-positive spermatogenic cells in the (A) control, (B) sham, (C) varicocele: TUNEL-positive cells (arrow), (D) varicocelectomy and (E) varicocelectomy and Quercetin groups. ${ }^{*} \mathrm{P}<0.05$ vs. control group; ${ }^{\#} \mathrm{P}<0.05$ vs. varicocele group; ${ }^{\wedge} \mathrm{P}<0.05$ vs. varicocelectomy group. TUNEL, terminal deoxynucleotidyl-transferase-mediated dUTP nick end labeling.

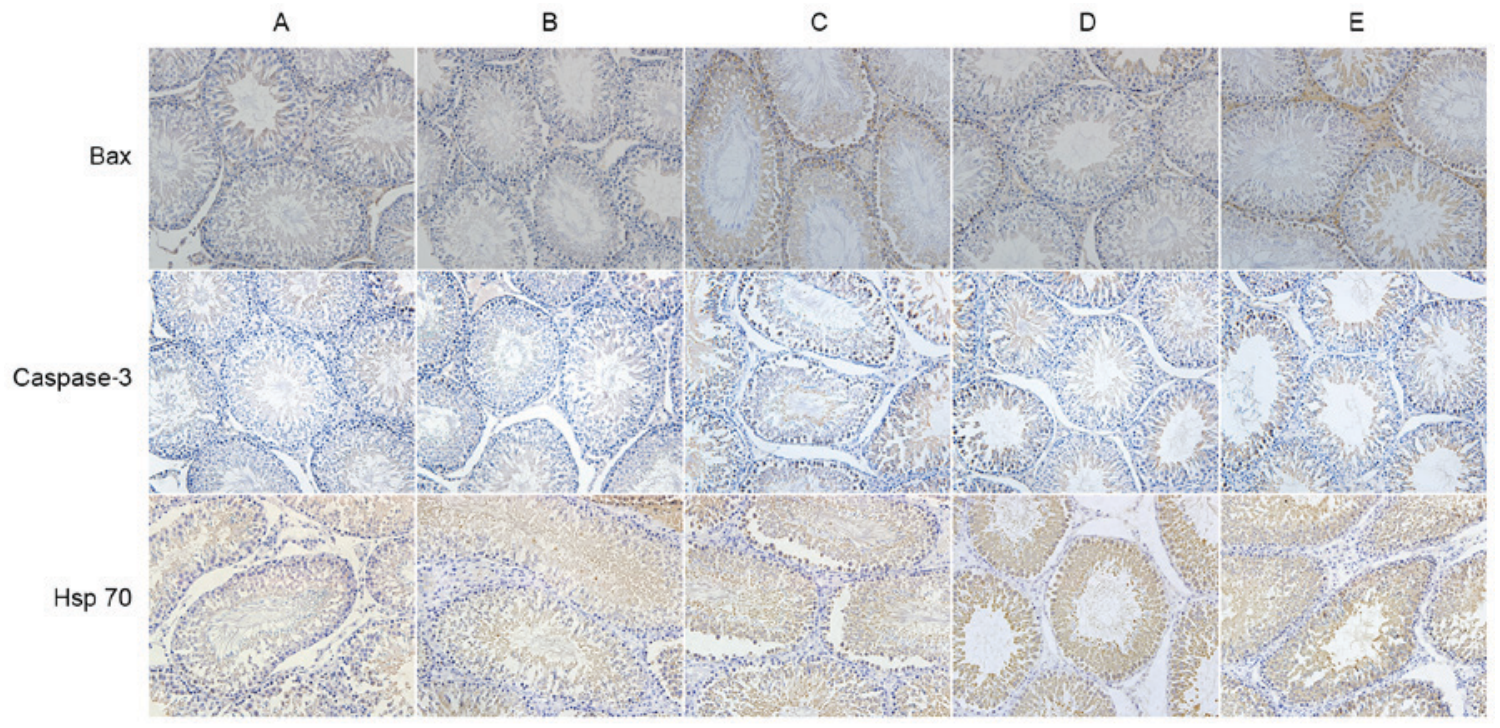

Figure 4. Immunohistochemical techniques were performed to evaluate the protein expression of Hsp70, caspase-3 and Bax (magnification, x200). Representative images of protein expression levels of Hsp70, Bax and caspase-3 in testis tissue in the (A) control, (B) sham, (C) varicocele, (D) varicocelectomy and (E) varicocelectomy and Quercetin groups. Hsp70, heat shock protein 70; Bax, Bcl-2-associated X protein.

group, the expression of these proteins was significantly increased in the left testis tissues, which appeared as yellow or brown granules in the cytoplasm and nucleus. Compared with the varicocele group, the expression of caspase- 3 and Bax was reduced in the varicocelectomy group, in contrast to the expression of Hsp70 (Fig. 4).

Western blot analysis. The protein expression of Bax, Bcl-2, cyt $c$ and cleaved caspase-3 was studied by western blotting. The results revealed higher levels of Bax expression in the varicocele group compared with the control and sham groups. However, varicocelectomy reduced the expression of Bax. In contrast, the expression levels of $\mathrm{Bcl}-2$ in the varicocele group were lower than those in the control and sham groups. After varicocelectomy, the expression levels of Bcl-2 were upregulated compared with the levels in the varicocele groups. The expression of cleaved caspase- 3 and cyt $c$ was increased in the varicocele group compared with the control and sham groups. The expression levels of cleaved caspase- 3 and cyt $c$ were significantly decreased in the varicocelectomy group, while the protective effects were inhibited by Quercetin (Fig. 5).

$R T-q P C R$ analysis. In order to investigate the mRNA expression levels of Hsp70, caspase-3 and 9, RT-qPCR analysis was performed. GAPDH was used as the housekeeping gene. The mRNA expression levels of Hsp70 and caspase-3 and 9 were 

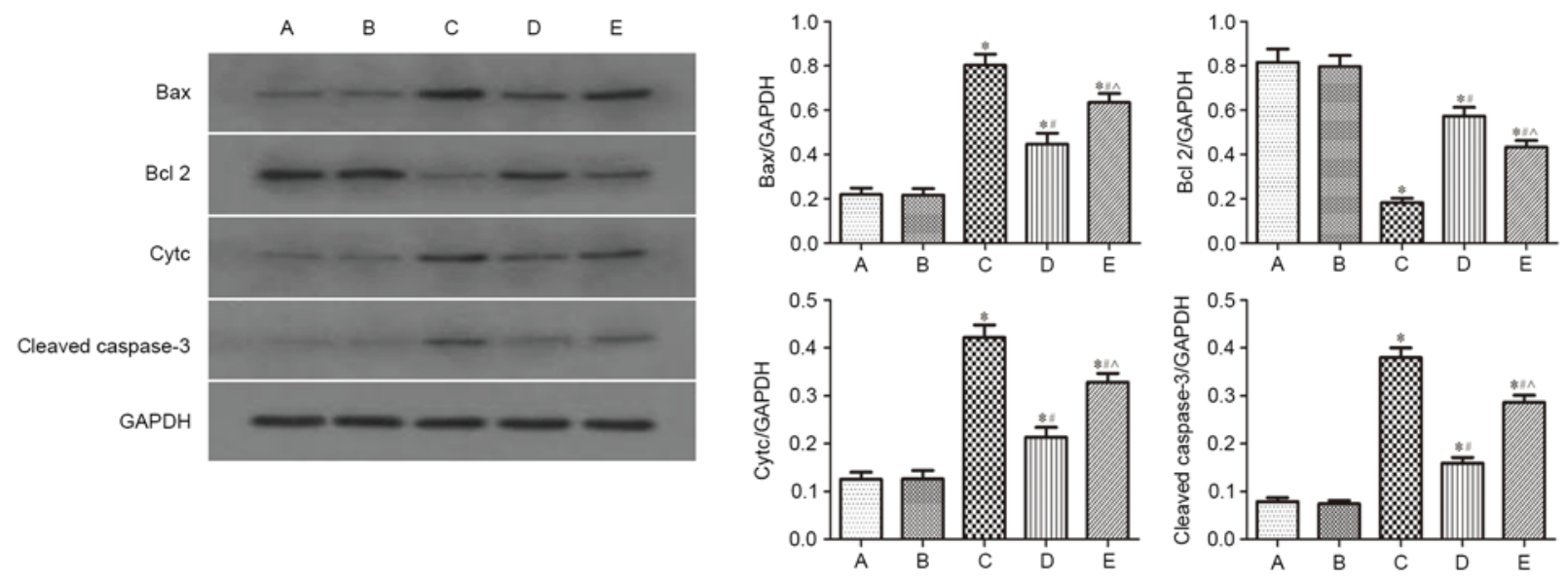

Figure 5. Protein expression levels of Bax and Bcl-2 were studied by western blot analysis. GAPDH was used as loading control. Representative western blot images and quantification of protein expression levels of $\mathrm{Bax}, \mathrm{Bcl}-2$, cyt $c$ and caspase-3 in rat testis. ${ }^{*} \mathrm{P}<0.05$ vs. control group, ${ }^{\#} \mathrm{P}<0.05$ vs. varicocele group, ${ }^{\wedge} \mathrm{P}<0.05$ vs. varicocelectomy group. A, control group; B, sham group; C, varicocele group; D, varicocelectomy group; E, varicocelectomy and Quercetin group. Bax, Bcl-2-associated X protein; Bcl-2, B-cell lymphoma 2; cyt $c$, cytochrome $c$.
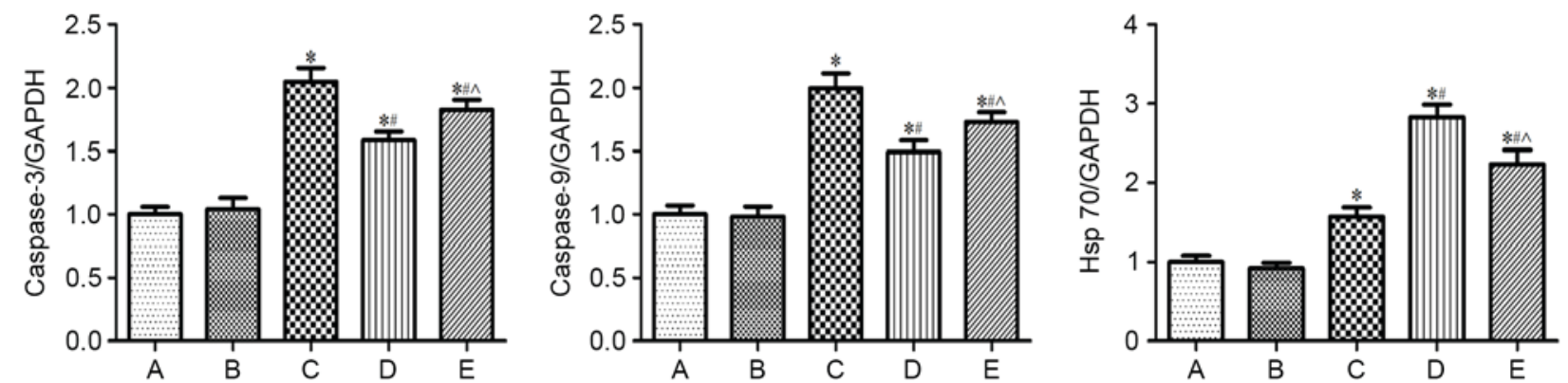

Figure 6. mRNA expression levels of Hsp70, caspase-3 and -9 relative to GAPDH were studied using reverse transcription-quantitative polymerase chain reaction. ${ }^{*} \mathrm{P}<0.05$ vs. control group, ${ }^{\#} \mathrm{P}<0.05$ vs. varicocele group, ${ }^{\wedge} \mathrm{P}<0.05$ vs. varicocelectomy group. A, control group; $\mathrm{B}$, sham group; $\mathrm{C}$, varicocele group; D, varicocelectomy group; E, varicocelectomy and Quercetin group. Hsp70, heat shock protein 70.

significantly higher in the varicocele group than in the control and sham groups. However, compared with the levels in the varicocele group, the mRNA expression levels of caspase-3 and 9 was reduced after varicocelectomy, whereas the expression levels of Hsp70 were increased. Quercetin inhibited the protective effect of varicocelectomy for caspase-3 and 9 levels (Fig. 6).

\section{Discussion}

Apoptosis is a process of programmed cell death based on a genetic mechanism that includes a range of cellular, morphological and biochemical changes (13). The process of apoptosis is regulated by a precise regulation of gene expression. It is known that two major pathways are involved in cell apoptosis: The intrinsic (or mitochondrial) and extrinsic (or death receptor) signal transduction pathways (14). The intrinsic pathway includes Bcl-2, caspase-9 and mitochondrial membrane permeability $(15,16)$. Several studies have demonstrated that certain apoptosis-activating factors lead to apoptotic signals after acting on cells. The mitochondrial permeability transition pore is then opened and prompts the release of cyt $c$, the delivery of caspase- 9 and changes the Bcl-2/Bax ratio, which activates caspase-3, leading to cell apoptosis (17-19). Bcl-2 is a family of proteins including pro-apoptotic factors (such as Bax) and anti-apoptotic factors (such as Bcl-2) (20). These factors have recently been observed to act as a pair of closely related apoptotic proteins. The $\mathrm{Bcl}-2 / \mathrm{Bax}$ ratio, as a regulator of spermatogenic cell apoptosis, determines whether apoptosis occurs in cells exposed to damage (21). Onur et al (22) reported that the $\mathrm{Bcl}-2 / \mathrm{Bax}$ ratio is significantly decreased in experimental varicocele rats. Additionally, caspases serve a key role in complex apoptotic mechanisms (23). Caspase-3 is an inactive zymogen in the cytoplasm, which can be stimulated by external apoptotic factors. In this case, caspase- 9 is activated, leading to the activation of caspase-3, which in turn activates cell apoptosis via a signal transduction pathway. Changes in cell shrinkage, chromatin condensation and DNA fragmentation will occur (24). Furthermore, caspase-3 is the convergence point of multiple apoptosis-activating signals, and its activation is a sign of irreversible commitment to cell apoptosis (25). It is widely accepted that apoptosis leads to testicular spermatogenesis dysfunction and male infertility subsequent to varicocele (26). In a previous study, it was demonstrated that 
varicocele resulted in histopathologic changes of the testes in an experimental rat model (27). According to Zheng et al (28), spermatogenesis and apoptosis could be significantly improved by varicocelectomy in varicocele-induced rats. The present study demonstrated that compared with the control and sham groups, the apoptosis of spermatogenic cells was increased in varicocele-induced rats, and the number of apoptotic cells were significantly reduced following the varicocelectomy, as demonstrated by the TUNEL assay. H\&E staining revealed that damage of testicular spermatogenic function was higher in the varicocele group than in the control and sham groups, and that varicocelectomy markedly ameliorated spermatogenic cells and seminiferous epithelium injury, which is consistent with the results of the TUNEL assay.

HSPs are a group of stress-responsive proteins that are widely expressed in a variety of organisms (8). Hsp70 is an inhibitor of apoptosis that has multiple regulatory roles in the cellular signal transduction pathways, and it is involved in the cytoprotection against cell apoptosis (29-31). Several studies have demonstrated that there is an association between Hsp70 and the mitochondrial pathway. Upregulation of the expression of the Hsp70 can induce anti-apoptotic effects by reducing mitochondrial swelling and permeability (32). As a critical negative regulator of the mitochondrial pathway, the Hsp70 inhibits the release of cyt $c$, thereby blocking the activation of caspase-9 and caspase-3. This is the main pathway where Hsp70 prevents apoptosis (33).

In the present study, the effects of varicocelectomy on Hsp70 expression and the apoptosis of the testicular spermatogenic cells via the mitochondrial signal transduction pathway were investigated. Immunohistochemistry, western blotting and RT-qPCR were performed in order to determine the exact mechanism. The Bax/Bcl-2 ratio and the expression levels of caspase-9, cyt $c$ and caspase-3 were all upregulated in rat testes upon the induction of varicocele compared with the control and sham groups. This result demonstrated that apoptosis in rats with varicocele is initiated by the mitochondrial signal transduction pathway. In a previous study, an increase of apoptosis in the testes of rats and patients with varicocele via the mitochondrial signal transduction pathway was reported (34), which is consistent with the present study. Quercetin, as an inhibitor of HSPs, inhibits protein expression and alters the transcription pattern, including HSP27, HSP70 and heme oxygenase-1 (35). After performing the varicocelectomy, Hsp70 expression was significantly increased compared with the varicocele group. However, the $\mathrm{Bax} / \mathrm{Bcl}-2$ ratio and the expression of caspase-9, cyt $c$ and caspase-3 were decreased, in addition to the apoptosis of spermatogenic cells. Quercetin treatment attenuated these protective effects of varicocelectomy, indicating that varicocelectomy attenuated apoptosis by increasing the expression of the Hsp70.

In conclusion, the present study demonstrated that varicocelectomy can decrease the $\mathrm{Bax} / \mathrm{Bcl}-2$ ratio and can reduce the expression of caspase-9, cyt $c$ and caspase- 3 via the mitochondrial signal transduction pathway. It also demonstrated that varicocelectomy can protect cells from apoptosis. Such protective effects of spermatogenesis and apoptosis against varicocele injury may be due to the induction of Hsp70 but further research is required to clarify the mechanism by which varicocelectomy upregulates the expression of Hsp70 in testes.

\section{References}

1. Sadek A, Almohamdy AS, Zaki A, Aref M, Ibrahim SM and Mostafa T: Sperm chromatin condensation in infertile men with varicocele before and after surgical repair. Fertil Steril 95: 1705-1708, 2011.

2. Fretz PC and Sandlow JI: Varicocele: Current concepts in pathophysiology, diagnosis, and treatment. Urol Clin North Am 29: 921-937, 2002.

3. Pasqualotto FF, Lucon AM, Hallak J, Góes PM, Saldanha LB and Arap S: Induction of spermatogenesis in azoospermic men after varicocele repair. Hum Reprod 18: 108-112, 2003.

4. Lee JD, Lu LY, Cheng WH and Jeng SY: Dysregulated apoptosis through the intrinsic pathway in the internal spermatic vein of patients with varicocele. J Formos Med Assoc 108: 612-618, 2009.

5. Tek M, Cayan S, Yilmaz N, Oğuz I, Erdem E and Akbay E: The effect of vascular endothelial growth factor on spermatogenesis and apoptosis in experimentally varicocele-induced adolescent rats. Fertil Steril 91: 2247-2252, 2009.

6. Barqawi A, Caruso A and Meacham RB: Experimental varicocele induces testicular germ cell apoptosis in the rat. J Urol 171: 501-503, 2004.

7. Fazlioglu A, Yilmaz I, Mete O, Kurtulus F, Parlakkilic O, Güctas $\mathrm{O}$ and Cek $\mathrm{M}$ : The effect of varicocele repair on experimental varicocele-induced testicular germ cell apoptosis. J Androl 29: 29-34, 2008.

8. De Maio A: Heat shock proteins: Facts, thoughts, and dreams. Shock 11: 1-12, 1999.

9. Simar D, Jacques A and Caillaud C: Heat shock proteins induction reduces stress kinases activation, potentially improving insulin signalling in monocytes from obese subjects. Cell Stress Chaperones 17: 615-621, 2012.

10. Khosravanian H, Razi M, Farokhi F and Khosravanian N: Simultaneous administration of dexamethasone and vitamin E reversed experimental varicocele-induced impact in testicular tissue in rats; correlation with Hsp70-2 chaperone expression. Int Braz J Urol 41: 773-790, 2015.

11. Feng HL, Sandlow JI and Sparks AE: Decreased expression of the heat shock protein hsp70-2 is associated with the pathogenesis of male infertility. Fertil Steril 76: 1136-1139, 2001.

12. Turner TT: The study of varicocele through the use of animal models. Hum Reprod Update 7: 78-84, 2001.

13. Vaux DL and Korsmeyer SJ: Cell death in development. Cell 96: 245-254, 1999.

14. Kerr JF, Wyllie AH and Currie AR: Apoptosis: A basic biological phenomenon with wide-ranging implications in tissue kinetics. Br J Cancer 26: 239-257, 1972.

15. Chipuk JE and Green DR: Do inducers of apoptosis trigger caspase-independent cell death? Nat Rev Mol Cell Biol 6: 268-275, 2005.

16. Lysiak JJ, Turner SD and Turner TT: Molecular pathway of germ cell apoptosis following ischemia/reperfusion of the rat testis. Biol Reprod 63: 1465-1472, 2000.

17. Feng Y, Lu Y, Lin X, Gao Y, Zhao Q, Li W and Wang R: Endomorphins and morphine limit anoxia-reoxygenation-induced brain mitochondrial dysfunction in the mouse. Life Sci 82: 752-763, 2008.

18. Cui J, Chen C, Lu H and Shen P: Modelling of the mitochondrial apoptosis network. Int J Bioinform Res Appl 4: 172-187, 2008.

19. Hasenjager A, Gillissen B, Müller A, Normand G, Hemmati PG, Schuler M, Dörken B and Daniel PT: Smac induces cytochrome $\mathrm{c}$ release and apoptosis independently from $\mathrm{Bax} / \mathrm{Bcl}-\mathrm{x}(\mathrm{L})$ in a strictly caspase-3-dependent manner in human carcinoma cells. Oncogene 23: 4523-4535, 2004.

20. Adams JM and Cory S: The Bcl-2 protein family: Arbiters of cell survival. Science 281: 1322-1326, 1998.

21. Liang H, Yu F, Tong Z, Yuan B and Wang C: Effect of ischemia post-conditioning on skeletal muscle oxidative injury, mTOR, Bax, Bcl-2 proteins expression, and HIF- $1 \alpha / \beta$-actin mRNA, IL-6/ $\beta$-actin mRNA and caveolin-3/ $\beta$-actin mRNA expression in ischemia-reperfusion rabbits. Mol Biol Rep 40: 507-514, 2013.

22. Onur R, Semerciöz A, Orhan I and Yekeler H: The effects of melatonin and the antioxidant defence system on apoptosis regulator proteins (Bax and Bcl-2) in experimentally induced varicocele. Urol Res 32: 204-208, 2004.

23. Said TM, Paasch U, Glander HJ and Agarwal A: Role of caspases in male infertility. Hum Reprod Update 10: 39-51, 2004. 
24. Arnoult D, Parone P, Martinou JC, Antonsson B, Estaquier J and Ameisen JC: Mitochondrial release of apoptosis-inducing factor occurs downstream of cytochrome $\mathrm{c}$ release in response to several proapoptotic stimuli. J Cell Biol 159: 923-929, 2002.

25. Zheng Y, Zhang X, Zhou J, Cheng F and Zhou B: Effects on the ipsilateral testis during progression of experimental varicocele in rat. Med Sci Monit 14: BR122-BR126, 2008.

26. Tanaka H, Fujisawa M, Tanaka H, Okada $H$ and Kamidono $S$ : Apoptosis-related proteins in the testes of infertile men with varicocele. BJU Int 89: 905-909, 2002.

27. Choi H, Kim KS and Kim KM: The effect of experimental varicocele on the testis of adolescent rats. J Urol 144: 499-501; discussion 512-513, 1990.

28. Zheng YQ, Zhang XB, Zhou JQ, Cheng F, Rao T and Yao Y: The effects of artery-ligating and artery-preserving varicocelectomy on the ipsilateral testes in rats. Urology 72: 1179-1184, 2008.

29. Li P, Nijhawan D, Budihardjo I, Srinivasula SM, Ahmad M, Alnemri ES and Wang X: Cytochrome c and dATP-dependent formation of Apaf-1/caspase-9 complex initiates an apoptotic protease cascade. Cell 91: 479-489, 1997.
30. Kwon YK and Hecht NB: Cytoplasmic protein binding to highly conserved sequences in the $3^{\prime}$ untranslated region of mouse protamine 2 mRNA, a translationally regulated transcript of male germ cells. Proc Natl Acad Sci USA 88: 3584-3588, 1991.

31. Eddy EM: Role of heat shock protein HSP70-2 in spermatogenesis. Rev Reprod 4: 23-30, 1999.

32. Liao W, Li X, Mancini M and Chan L: Proteasome inhibition induces differential heat shock protein response but not unfolded protein response in HepG2 cells. J Cell Biochem 99: 1085-1095, 2006.

33. Rokutan K: Role of heat shock proteins in gastric mucosal protection. J Gastroenterol Hepatol 15 (Suppl): D12-D19, 2000.

34. Lee JD, Lee TH, Cheng WH and Jeng SY: Involved intrinsic apoptotic pathway of testicular tissues in varicocele-induced rats. World J Urol 27: 527-532, 2009.

35. Manwell LA and Heikkila JJ: Examination of KNK437- and quercetin-mediated inhibition of heat shock-induced heat shock protein gene expression in Xenopus laevis cultured cells. Comp Biochem Physiol A Mol Integr Physiol 148: 521-530, 2007. 